Société d'histoire de la révolution de 1848 et des

révolutions du XIXe siècle

52 | 2016

Chrononymes. Dénommer le siècle

\title{
Fin de siècle
}

"Fin de siècle"

«Fin de siècle»

\section{Christophe Charle}

\section{Q OpenEdition}

\section{Journals}

Édition électronique

URL : http://journals.openedition.org/rh19/4995

DOl : $10.4000 /$ rh 19.4995

ISSN : $1777-5329$

Éditeur

La Société de 1848

\section{Édition imprimée}

Date de publication : 1 juin 2016

Pagination : 103-117

ISSN : 1265-1354

\section{Référence électronique}

Christophe Charle, "Fin de siècle », Revue d'histoire du XIXe siècle [En ligne], 52 | 2016, mis en ligne le

01 juin 2019, consulté le 03 mai 2019. URL : http://journals.openedition.org/rh19/4995 ; DOI:

10.4000/rh19.4995 


\section{CHRISTOPHE CHARLE}

\section{Fin de siècle}

La singularité du chrononyme «fin de siècle» tient à la précocité de son autodéfinition. D'ordinaire, la plupart des expressions qualifiant des périodes sont rétrospectives, qu'on pense à la «Belle époque» ou aux trop fameuses "Trente Glorieuses " ${ }^{1}$. Les contemporains ont beau dénoncer eux-mêmes l'artifice qui consiste à identifier l'évolution d'un siècle aux phases d'essor, d'apogée et de déclin d'un corps organique qui traverse les "âges de la vie", plus on approche des décennies fatidiques, plus la thématique s'affirme et ceux qui tentent de la contester ou de proposer une autre vision du temps historique doivent recourir à des procédés rhétoriques défensifs. Cette mode terminologique aurait donc dû s'épuiser avec le moment qui l'a vu naître. Pourtant force est de constater qu'elle est toujours vigoureuse et abondamment sollicitée comme étiquette pour de nombreux travaux contemporains prenant ainsi la force d'une évidence malgré ses fragilités originelles. C'est à explorer ce paradoxe que nous nous emploierons ici. Au-delà du cas singulier, il peut fournir en effet des enseignements sur les défauts récurrents des modes de périodisation qui font souvent écran à la compréhension d'un moment par les problématiques parasites inconscientes qu'ils induisent ${ }^{2}$.

$*$

Avatars DU « SIÈCLE $»^{3}$

Pour comprendre l'apparition, la disparition, puis la résurrection de «fin de siècle", il faut remonter assez loin dans le temps et ne pas se contenter

1. $C f$. la contribution de Dominique Kalifa dans ce même volume sur la "Belle époque», et Rémy Pawin, "Retour sur les "Trente Glorieuses" et la périodisation du second XXe siècle», Revue d'histoire moderne et contemporaine, 2013, $\mathrm{n}^{\circ}$ 60-1, p. 155-175.

2. Stéphane Gibert, Jean Le Bihan, Florian Mazel [dir.], «Découper le temps. Actualité de la périodisation en histoire", Atala, n 17, 2014, en particulier l'avant-propos de Stéphane Gibert, "Les enjeux renouvelés d'un problème fondamental : la périodisation en histoire» p. 7-31, et ma postface "Discordance des temps, dénivellations spatiales et périodisation, les défis de l'homo historicus", p. 325-334.

3. Cf. sur ce point, Jean Leduc, Les Historiens et le temps. Conceptions, problématiques, écritures, Paris, Seuil, 1999, p. 97-99. 
d'examiner, comme on pourrait le croire, uniquement les décennies terminales du XIX ${ }^{\mathrm{e}}$ siècle, sa période d'éclosion et de consécration postérieure par les travaux savants sur lesquels nous reviendrons plus loin. Il faut partir du coup de force symbolique fondateur de Voltaire concernant le «siècle» comme unité chronologique pertinente. En 1751, l'auteur de Candide arrache le mot siècle à ses connotations religieuses péjoratives et en fait avec Le siècle de Louis $X I V$ le premier chrononyme érigé en norme d'appréciation pour construire les représentations de longue durée des périodes historiques (les précédents découpages canonisés antérieurs comme «Moyen Age», "Antiquité» embrassaient plusieurs "siècles»). C'est l'ouverture de ce livre sur le XVII" siècle appréhendé globalement qui change le statut d'un mot banal tout en élevant une statue au grand roi :

«Mais quiconque pense, et, ce qui est plus rare, quiconque a du goût, ne compte que quatre siècles dans l'histoire du monde. Ces quatre âges heureux sont ceux où les arts ont été perfectionnés et qui, servant d'époque à la grandeur de l'esprit humain sont l'exemple de la postérité.

Le premier de ces siècles, à qui la véritable gloire est attachée, est celui de Philippe et d'Alexandre, ou celui des Périclès, des Démosthène, des Aristote, des Platon, des Apelle, des Phidias, des Praxitèle; et cet honneur a été renfermé dans les limites de la Grèce; le reste de la terre connue alors était barbare.

Le second âge est celui de César et d'Auguste, désigné encore par les noms de Lucrèce, de Cicéron, de Tite-Live, de Virgile, d'Horace, d'Ovide, de Varron, de Vitruve.

Le troisième est celui qui suivit la prise de Constantinople par Mahomet II. Le lecteur peut se souvenir qu'on vit alors en Italie une famille de simples citoyens faire ce que devaient entreprendre les rois de l'Europe. Les Médicis appelèrent à Florence les savants, que les Turcs chassaient de Grèce; c'était le temps de la gloire de l'Italie. Les beaux-arts y avaient déjà repris une vie nouvelle; les Italiens les honorèrent du nom de vertu, comme les premiers Grecs les avaient caractérisés du nom de sagesse. Tout tendait à la perfection. (...)

Le quatrième siècle est celui qu'on nomme le siècle de Louis XIV, et c'est peut-être celui des quatre qui approche le plus de la perfection. Enrichi des découvertes des trois autres, il a plus fait en certains genres que les trois ensemble ${ }^{4}$.»

La notion de siècle comme époque spécifique aux caractères originaux naît avec cette périodisation de la civilisation par Voltaire. Elle implique, comme le montre ce début du Siècle de Louis XIV, l'alternance de rares temps glorieux et de longs temps obscurs ou de décadence dominant l'histoire des hommes. En réaction, les autres tenants des Lumières (et lointainement jusqu'à

4. Voltaire, Le siècle de Louis XIV (1751), édition René Pomeau, in Euvres historiques, Paris, Gallimard, Bibliothèque de la Pléiade, 2000, p. 616 et 617. 
Michelet) vont construire contre la vision voltairienne statique, la thématique d'une avancée continue dans leur propre siècle (siècle des Lumières) qui rompt avec la discontinuité fataliste entre siècles glorieux et siècles obscurs. Malgré la trouble décennie finale du XVIII ${ }^{\mathrm{e}}$ siècle, cette nouvelle dynamique des siècles trouve son expression synthétique dans le célèbre essai de Condorcet, Esquisse d'un tableau historique des progrès de l'esprit humain (1794) où il divise l'histoire en "époques» au nombre de dix dont une dessinant l'avenir; chacune apporte sa pierre au progrès global.

En revanche, les adversaires de la Révolution et, plus généralement, des Lumières n'ont pas recouru pour autant à la thématique logique de la «fin de siècle» qui aurait renoué avec la discontinuité voltairienne. Ils ont préféré convoquer, on le sait, une rhétorique renouvelée de la vision religieuse de l'histoire où l'orgueil humain, héritage du péché originel, que ce soit celui du grand homme (Napoléon en premier lieu) ou des hommes révoltés des foules révolutionnaires est puni par la loi divine à travers une série de catastrophes qui obligent la France à rentrer dans l'ordre ancien : terreur, guerre civile, invasion, occupation, restauration. Mais comme cette restauration a fait long feu du moins en France, dès les années 1820 et plus encore après 1830, chez les libéraux, les saint-simoniens comme chez les républicains, la conception du progrès héritée de Condorcet a relancé l'identification du nouveau siècle avec un mouvement ascendant. Il s'applique maintenant avec les mutations techniques visibles dans la vie courante à des domaines plus vastes que les lettres, les arts ou les sciences chers aux Encyclopédistes 5 . Au milieu du XIX ${ }^{e}$ siècle, cette vision positive du nouveau siècle, en dépit des critiques émanant des milieux conservateurs ou catholiques intransigeants ou de minorités intellectuelles décalées et ironiques, acquiert peu à peu la force du lieu commun en tout cas dans la partie la plus visible des publicistes et au sein du public bourgeois qui les lit, au point de susciter l'agacement méprisant des intellectuels et artistes les moins conformistes, Baudelaire et Flaubert en têté .

Cet éloge du temps présent est régulièrement réactivé à l'occasion notamment des bilans dressés lors des expositions universelles toujours dans le même esprit de comparaison dans le temps et dans l'espace au profit de la France. Le gouvernement impérial, en 1855 comme en 1867, en convoquant le monde entier à Paris, peut se targuer des réussites parisiennes en matière d'urbanisme, des progrès industriels et techniques qui facilitent le quotidien de tous et d'un rayonnement artistique international encore inentamé. 2011.

5. Voir Christophe Charle, Discordance des temps. Une brève histoire de la modernité, Paris, Colin,

6. Cette cohabitation entre mainstream et humeur critique date des lendemains de 1830 comme le montre José-Luis Diaz ("Comment 1830 invente le XIX ${ }^{e}$ siècle») ou Alain Corbin ("Le XIX ${ }^{e}$ siècle ou la nécessité de l'assemblage») in L'invention du XIX siècle, textes réunis par A. Corbin, P. Georgel, S. Guégan, S. Michaud, M. Milner, N. Savy, Paris, Klincksieck/Presses de la Sorbonne nouvelle, 1999 respectivement p. 177-193 et p. 153-159. Elle facilitera le retournement de l'humeur général après 1870. 
Pourtant la fin des années 1860 amorce déjà un tournant comme l'indiquent certains passages de l'introduction au rapport général sur l'Exposition universelle de 1867. Le commissaire général, Michel Chevalier y perçoit des signes inquiétants qui ne peuvent s'accorder avec la thématique du progrès inéluctable et surtout de l'hégémonie indéfinie de l'Europe sur le reste du monde :

«L'Europe qui se considère comme la représentation la plus élevée du genre humain, l'Europe qui, à l'heure actuelle, possède les arts utiles et les beaux-arts, attributs distinctifs et signes caractéristiques de la civilisation, l'Europe dont les enfants réunis dans l'enceinte de l'Exposition, semblaient prêts à se serrer dans les bras les uns des autres, offre bien plus l'aspect d'un camp que celui d'un groupe de communautés d'hommes industrieux et éclairés, honorant Dieu, aimant leurs semblables, jaloux de faciliter le progrès universel et individuel par le développement de la liberté générale et des libertés particulières.

Si loin qu'on remonte dans l'histoire, on ne retrouvera jamais une pareille collection d'hommes armés, un pareil amoncellement d'instruments de guerre ${ }^{7}$.»

Ces appréciations pessimistes frappent d'autant plus qu'elles concluent le volume. Elles contrastent avec la tonalité générale positive du rapport et renvoient explicitement aux conflits récents qui ont ensanglanté l'Europe, en particulier la victoire de la Prusse sur l'Autriche à Sadowa en 1866. Elles débouchent sur l'annonce possible d'un déclin de la civilisation incarnée par l'Europe et de manière superlative par la France (l'échec de l'expédition du Mexique l'année même où sont écrites ces lignes en serait le premier indice). Ce pronostic est d'autant plus inquiétant qu'il émane d'un haut responsable qui depuis sa jeunesse saint-simonienne a toujours eu foi dans un progrès indéfini rompant avec les malédictions des siècles précédents.

En fait cette thématique d'une fin de siècle possiblement inquiétante avait été esquissée chez des publicistes marginaux mais dont les lieux communs vont être réactivés dans les décennies suivantes. En 1852 par exemple, on pouvait lire sous la plume d'un auteur conservateur Eugène de Montlaur dans De l'Italie et de l'Espagne:

«Le début du XIX ${ }^{e}$ siècle a été plein d'éclat et de grandeur.

Le XVIII ${ }^{e}$ siècle s'était abîmé dans une nuit profonde. Il y avait eu, vers la fin, comme un universel écroulement; et sur les ruines amoncelées des lois, des mours, des croyances, comme une mêlée générale des peuples du continent. On combattait ici au nom de l'ordre social attaqué jusque dans ses fondements; là, au nom des confuses théories qu'une démagogie furieuse,

7. Exposition universelle de 1867, Rapports du jury international introduction par M. Michel Chevalier, Paris, Imprimerie administrative Paul Dupont, 1868, p. DXII. Téléchargeable sur http:// cnum.cnam.fr/PDF/cnum_8XAE149.1.pdf. 
excitée par d'insatiable ambitions, expliquait sur les places publiques avec des torches incendiaires et l'échafaud. Cette fin de siècle ressemble à ces fantasques et grandioses tableaux du peintre anglais Martins, comme eux elle donne le vertige et fait douter le penseur de la marche progressive de l'humanité ${ }^{8}$.»

Ce schéma binaire qui voudrait que les débuts et les fins de siècle obéissent à des orientations opposées est donc présent comme représentation bien avant qu'il n'en soit fait un usage intensif à partir des années 1880. En 1855, Eugène Huzar prend le contrepied de la vision dominante en annonçant non le progrès mais «la fin du monde par la science ${ }^{9}$ ": le progrès technique à mesure qu'il s'accélère causera des catastrophes et des dégâts naturels de plus en plus grands. Ces ombres au tableau du siècle triomphant vont s'étendre encore un peu plus dans les décennies suivantes.

\section{TRENTE ANS AVANT}

La «fin de siècle» comme représentation inquiétante commence donc précocement en France, trente ans avant même la fin officielle du siècle. Des thématiques qui seront déclinées à satiété au cours des années 1890 surgissent dès l'orée des années 1880, voire dans les années 1870 à l'occasion des amères réflexions sur la défaite face à l'Allemagne ${ }^{10}$. Cette précocité renvoie à la coïncidence de trois phénomènes indépendants mais qui vont produire leurs effets cumulatifs à l'apogée du thème de la décadence ou de la dégénérescence associé à l'idée de "fin de siècle». Le premier est l'incertitude politique qui dure de 1871 à 1878 et l'hostilité d'une fraction importante des intellectuels liés à l'Empire ou à l'Ordre moral face à l'avènement du nouveau régime. Le plus célèbre est évidemment Taine, principale autorité du champ intellectuel avec Renan jusqu'à sa mort en $1893^{11}$. Dans ses Notes sur l'Angleterre (1872) comme dans Les Origines de la France contemporaine (1875-1893) il développe la thématique du déclin national par un jeu de miroirs avec l'Angleterre, préservée des révolutions. Il établit un parallèle entre la fin du XVIII ${ }^{\mathrm{e}}$ siècle, l'époque révolutionnaire, et le moment où il écrit

8. Eugène de Montlaur, De l'Italie et de l'Espagne, études historiques et critiques, Paris, Garnier, 1852 , p. II.

9. Eugène Huzar, La Fin du monde par la science, introduction de Jean-Baptiste Fressoz, Alfortville, Ere, 2008. Cf. aussi sur ces "dégâts du progrès" avant la lettre, Jean-Baptiste Fressoz, L'Apocalypse joyeuse. Une histoire du risque technologique, Paris, Seuil, 2012 et François Jarrige, Technocritiques. Du refus des machines à la contestation des technosciences, Paris, La Découverte, 2014.

10. Sur les réflexions précoces sur la décadence française dès 1870-1871, cf. Christophe Charle, "Trois écrivains face à une autre "étrange défaite" : Goncourt, Flaubert et Zola et la guerre de 1870 ", in A. Betz et S. Martens [dir.], Les Intellectuels et l'occupation (1940-44), Paris, Autrement, 2004, p. 14-37.

11. Cf. Christophe Charle, Paris fin de siècle. Culture et politique, Paris, Seuil, 1998, chapitre 3 et Nathalie Richard, Hippolyte Taine. Histoire, psychologie, littérature, Paris, Garnier, 2013. 
qu'il assimile au retour des "jacobins", ancêtres supposés des républicains radicaux hissés au pouvoir après 1879 . Ce livre connait un large succès chez les lettrés et à l'étranger (par exemple chez Nietzsche qui le cite) et prépare les esprits aux thématiques "fin de siècle» et «décadentes»"

Le second facteur incitant à l'humeur "décliniste» est le climat économique général déprimé après le krach boursier de 1882, les difficultés agricoles et l'inquiétude liée à la faiblesse démographique française en regard de l'essor de la population allemande et des pays "anglo-saxons" attesté par le développement de leurs colonies de peuplement. Le premier aspect concerne toute l'Europe, mais se traduit par le recul économique relatif de la position française face à l'Allemagne ou à l'Angleterre et bientôt aux puissances extra-européennes comme les Etats-Unis. Le discours néo-saint-simonien ou libre-échangiste qui avait dominé sous le Second Empire cède devant un discours défensif, protectionniste, y compris dans des milieux autrefois libéraux. Le projet colonial de la nouvelle République de Jules Ferry s'en sert comme argument a contrario : puisque les marchés européens échappent à la France il faut en construire de nouveaux dans des «espaces vierges» et protégés d'Afrique et d'Asie.

Le troisième motif central et le plus important dans la thématique fin de siècle est celui d'une crise morale, fondement secret des déclins matériels précédents : le pessimisme, l'exacerbation des passions et des excitations nerveuses seraient produits par la civilisation moderne. Cette crise touche en premier lieu les élites urbaines des grandes villes et particulièrement les milieux artistiques et littéraires les plus exposés à cette modernité maladive dans la lutte de concurrence de tous contre tous que diffuse la vulgarisation du thème darwinien de la lutte pour la vie. Ce schème interprétatif est, lui aussi, précoce comme l'indique cet extrait de 1882 sous la plume d'un critique dramatique peu enclin d'ordinaire aux considérations philosophiques :

«Dernièrement, quelqu'un faisait remarquer devant moi que nos artistes, maintenant si rangés et si sérieux, lorsqu'ils observent la société moderne, n’y voient plus que des fous, des exaltés, des artistes en un mot. C'est le monde retourné. On est tenté de leur dire. Où sont-ils donc, vos malades? Montreznous, vos détraqués! Dans cette fin de siècle, qui pour quelques gouttes de morphine voudrait se donner les airs d'une somnambule, la grande et unique névrose ne serait-elle pas la cupidité 13" $^{\text {" }}$

12. Dans Le Disciple (1889) Paul Bourget met en scène un philosophe qui ressemble à Taine et qu'il rend responsable du cynisme et du manque de sens moral de la jeunesse décadente contemporaine.

13. Arnold Mortier, Les Soirées parisiennes, Paris, Dentu, 1882, p. X. 
La même année, un romancier populaire Dubut de Laforest cite les mêmes lieux communs sur les névroses contemporaines dans la préface d'un roman au titre parlant Tête à l'envers, situé dans la «bonne société»:

«Et à cette heure où tout le monde dit que les cerveaux se détraquent, que la névrose nous talonne et que l'humanité touche à sa fin, ce ne serait pas un mince honneur pour ce petit roman que d'avoir remis en question le problème si grave et encore non résolu du libre arbitre.

En vérité, il serait curieux pour cette fin de siècle envahie par un formidable désir d'expérimentation, de savoir si toutes les femmes qui tombent sont bien toujours maîtresses de rester debout contre vents et marée... ${ }^{14}$ »

Un sondage réalisé avec la fonction recherche sur l'expression «fin de siècle» dans les volumes numérisés dans Gallica confirme que ces associations d'idée précoces ne relèvent pas du hasard. Une constellation de vocables négatifs surgit quasi automatiquement sous la plume des auteurs les plus divers quand ils recourent à l'expression «fin de siècle", au point que ce sont les rares tenants d'un reste d'optimisme en cette fin de siècle qui sont en position défensive face à cette nouvelle opinion dominante. En voici quelques exemples :

Léon Bloy (1884) : «Cette fin de siècle redoutable et chargée de mystère, comme la plupart des fins de siècle ${ }^{15}$.»

Dubut de Laforest (1884) : « en cette fin de siècle, un peu troublée, où tant de cerveaux se détraquent ${ }^{16}$.»

Léon Bloy (1886) : «La déliquescente psychologie littéraire de cette fin de siècle ${ }^{17} . "$

Edouard Drumont (1889) : «les mornes tristesses de cette fin de siècle ${ }^{18}$.»

L'étude exhaustive par Marc Angenot des publications de l'année 1889, permet de confirmer que ces associations d'idées caractéristiques se retrouvent dans tous les types de discours et dans tous les supports en attendant que d'autres les remplacent en approchant de 1900. On voit toutefois qu'ils sont bien antérieurs à l'année choisie pour son enquête en fonction de la coïncidence avec le centenaire de 1789 et des possibles effets d'écho entre «fins de siècle $»^{19}$.

14. Jean-Louis Dubut de Laforest, Tête à l'envers, Paris, Charpentier 1882, p. VI-VII (préface datée de mai 1882).

15. Léon Bloy, Propos d'un entrepreneur de démolitions, Paris, Tresse, 1884, p. 271.

16. Jean-Louis Dubut de Laforest, Une Livre de sang, Paris, Dentu 1884, p. 274.

17. Léon Bloy, Le Désespéré, Paris, A Soirat, 1886, p. 38.

18. Edouard Drumont, La Fin d'un monde, Paris, Savine 1889, p. 115.

19. Marc Angenot, 1889. Un état du discours social, Longueuil, Québec, Editions du Préambule, 1989, notamment p. 373-407. 
Face à ces thématiques négatives, les tenants fervents du progrès se retrouvent isolés dans des registres militants ou des discours officiels peu audibles et obligés de se situer face aux pessimistes de la décadence. Ainsi dans cette phrase conclusive des souvenirs d'un ancien recteur aux balancements incertains quant au diagnostic :

«S'il était vrai que la santé morale d'un pays se mesure au prestige de l'autorité qui est en haut dans le pouvoir, à la force qui est en bas dans la famille, notre fin de siècle serait bien malade, quelque rayonnement que projettent sur elle les merveilles de la science et les splendeurs de l'art ${ }^{20}$."

Trois ans plus tôt, Eugène Melchior de Voguë avait pourtant tenté de démonter le paralogisme sur lequel repose l'expression "fin de siècle" et les déductions fallacieuses quant à l'évolution morale qu'elle permettait de répandre :

"On se laisse volontiers abattre par ce mot fatidique une fin de siècle. C'est un leurre. Le siècle commence toujours pour ceux qui ont vingt ans. Nous avons divisé le temps en périodes artificielles, nous les comparons aux décours d'une existence humaine; la force créatrice de la nature se soucie peu de nos calculs; elle pousse sans relâche des générations dans le monde, elle leur confie un nouveau trésor de vie sans regarder l'heure à notre cadran ${ }^{21}$.»

Les rares professeurs d'optimisme ou d'énergie n'y peuvent rien. Tout pousse en sens inverse : l'atmosphère générale de crise évoquée précédemment, la vogue chez les écrivains et les intellectuels du pessimisme à la Schopenhauer qu' on commence à traduire ${ }^{22}$, la reprise par provocation des termes "décadence» ou "décadents» par la jeunesse littéraire à la recherche d'étiquettes nouvelles pour se distinguer des aînés dans la "bataille littéraire »" l'incapacité des nouvelles élites de la République à donner une image positive de leur action en dépit des réformes engagées antérieurement. L'amplification et l'extension du chrononyme "fin de siècle" s'opèrent par le détour de sa vulgarisation à l'étranger dans le livre à succès de Max Nordau, Entartung (1892), traduit par Dégénérescence dans l'édition française de 1894.

Louvrage bientôt adapté dans les principales langues européennes (néerlandais, 1893; italien 1893; français 1894; anglais, 1895) confère à la France une place centrale, et sa première partie est intitulée précisément «Fin de siècle». Le premier chapitre "Crépuscule des peuples" érige la France en paradigme de tous les maux «fin de siècle» résumés précédemment : p. 397.

20. Ad. Mourier, Notes et souvenirs d'un universitaire, Paris, imprimerie Jacob, 1889, conclusion,

21. Eugène Melchior de Voguë, Le roman russe, Paris, Plon, 1886, p. LII.

22. René-Pierre Colin, Schopenhauer en France : un mythe naturaliste, Lyon, PUL, 1979, p. 130-131.

23. Noël Richard, À l'aube du symbolisme, Paris, Nizet, 1961. 
«Fin de siècle» est français, car c'est la France qui, la première a eu conscience de l'état d'esprit que l'on dénomme ainsi. Le mot a volé à travers les deux mondes et a trouvé accès dans toutes les langues cultivées. C'est la preuve qu'il répondait à un besoin. L'état «fin de siècle» des esprits se rencontre aujourd'hui partout; mais il n'est, dans beaucoup de cas que l'imitation d'une mode étrangère tenue pour distinguée, et n'a rien d'organique. C'est dans son pays d'origine qu'il se présente de la façon la plus authentique; et Paris est l'endroit désigné pour l'observer dans ses manifestations variées ${ }^{24}$."

Si Max Nordau a synthétisé dans son best-seller les principaux thèmes du chrononyme et en a fait un mot de passe européen, il n'est en rien un inventeur, on l'a vu. Il profite de sa position de passeur entre cultures : juif hongrois détaché de sa religion d'origine, de culture allemande, il vit à Paris depuis 1880 et sert de correspondant à des journaux germaniques. Cette situation est idéale pour vulgariser les thèmes à la mode d'un pays à l'autre, jouer sur le monopole d'information que lui procure son long séjour à Paris où il a achevé ses études de médecine. Sous les dehors apparemment "objectifs» d'une enquête nosographique ${ }^{25}$, il propose le portrait parfaitement négatif de nombreuses personnalités parisiennes célèbres ou moins célèbres, renforcées par quelques novateurs étrangers (Wagner, Tolstoï), tous pris comme témoins et acteurs de la dégénérescence fin de siècle. Il reprend les expressions qui font l'air du temps du Paris littéraire et artistique des années 1880; celles qui ont cours dans le monde des boulevards et des théâtres ou dans les cafés fréquentés par la bohème du quartier latin et sont ressassées comme on l'a vu sous toutes les formes (anecdotes et faits divers, articles de journaux, de revues, essais, personnages typés de romans parisiens comme $\grave{A}$ Rebours (1884) ou Là-bas de Huysmans (1891), pièces de théâtre) depuis plus d'une dizaine d'années ${ }^{26}$. Abandon des traditions, fin d'un monde, obsolescence rapide des idées, souci de la surenchère, goût pour la déviance sous toutes ses formes, snobisme suiviste, tous ces traits de la fin de siècle selon Nordau sont concentrés dans la mince élite d'avant-garde parisienne qui lance les modes, profitant de la caisse de résonance d'une presse assoiffée de polémiques (voir l'Enquête sur l'évolution littéraire de Jules Huret, Paris, Charpentier, 1891),

24. Max Nordau, Dégénérescence, rééd., Lausanne, L’Age d'homme, 2010, p. 31 (première édition Berlin, Duncker \& Humblot, 1892, traduction française : Paris, Alcan, 1894).

25. L'emploi d'un vocabulaire pseudo médical qu'il emprunte à sa formation première ou à quelques auteurs connus (Morel, Lombroso, etc.) et la dédicace élogieuse à Cesare Lombroso sont autant de signes de ce double jeu littéraire et para scientifique qu'un autre outsider, auteur de bestsellers sur l'air du temps, Gustave Le Bon, pratique au même moment et sur des thèmes similaires.

26. L'auteur avoue lui-même comment il a construit son propos à partir des publications récentes : "Le plus sûr moyen de savoir ce qu'on entend par «fin de siècle» est de passer en revue une série de cas où ce mot a été employé. Ceux que nous allons citer ici sont empruntés aux journaux et aux livres français des deux dernières années." (Max Nordau, op. cit., p. 33). 
du cosmopolitisme d'une capitale qui donne encore le ton à des avant-gardes similaires dans d'autres pays :

«Une période de l'histoire touche manifestement à son terme, et une autre s'annonce. Toutes les traditions sont traversées d'une déchirure, et demain ne semble pas vouloir se rattacher à aujourd'hui; ce qui existe chancelle et s'écroule, et on le laisse s'affaler parce qu'on en est las et que l'on ne croit pas sa conservation digne d'un effort ${ }^{27}$.»

\section{RenOUVEAU DE LA «FIN DE SIÈCLE»}

En dépit de l'hostilité suscitée en France par le miroir négatif tendu par Dégénérescence lors de sa publication chez Alcan, les ouvres des auteurs attaqués nommément par Max Nordau comme symboles de la décadence du pays (au premier chef Zola et les naturalistes, certains poètes symbolistes) prolongent largement la thématique décadente et fin de siècle jusqu'à l'année 1900 , voire au-delà. Cette dernière était pourtant censée mettre un terme (puisqu'un nouveau siècle commence) au processus de dérèglement, d'autant qu'elle coïncide avec une nouvelle Exposition universelle vouée à ouvrir le $\mathrm{xx}^{\mathrm{e}}$ siècle, à faire oublier les combats de l'affaire Dreyfus et à renouer avec la thématique du progrès d'une humanité réconciliée dans la ville lumière.

De fait, les principaux romanciers proposent encore dans les dernières années de la décennie 1890 voire au début du xx $x^{\mathrm{e}}$ siècle, des tableaux décourageants des évolutions sociales, culturelles ou politiques qui n'ont rien à envier aux diagnostics pessimistes des essayistes comme Le Bon dans la Psychologie des foules (1895) ou des sociologues comme le Durkheim du Suicide (1896). La trilogie des Trois villes de Zola se clôt sur deux portraits décadents des capitales de la civilisation, celui de la cohabitation difficile entre l'ancienne Rome papale et la nouvelle Rome monarchique (Rome, 1896), celui du Paris (1898) des attentats anarchistes et du scandale de Panama transposé sous des clés transparentes pour les contemporains. La trilogie des Déracinés de Barrès (1897) prolongé par L'Appel au soldat (1900) et Leurs figures (1902) instruit le procès de la corruption de la vie publique dans la république des scandales, du boulangisme au panamisme. Les romans inspirés par l'affaire Dreyfus où les auteurs règlent les comptes non encore soldés par le compromis boiteux du procès de Rennes et de la grâce présidentielle (1899) entretiennent le souvenir de la crise fin de siècle bien au-delà de 1900 , ce que la querelle anticléricale ou la poussée des conflits sociaux dans les années 1900 amplifie encore ${ }^{28}$. Bref la fin de siècle et son cortège de décadence, de névrose

27. Ibidem, p. 35 .

28. Christophe Charle, Paris fin de siècle, op. cit., chapitre 7; Jacqueline Lalouette, «L'affaire Dreyfus dans le roman français", Revue historique, 1999, n 123, p. 555-576; Assia Kettani, De 
et d'incertitudes politiques et sociales n'en finissent pas de finir, du moins dans l'espace des représentations littéraires et journalistiques les plus diffusées. "L'esprit fin de siècle» fait de cynisme et de transgression des tabous moraux éclate aussi dans la crudité des confessions du Journal d'une fermme de chambre d'Octave Mirbeau où la femme de chambre d'origine bretonne, déniaisée par des maîtres parisiens très "fin de siècle», finit par incarner une certaine vertu face à l'hypocrisie de bourgeois provinciaux étriqués mais tout aussi obsédés par l'argent ou le sexe que les Parisiens ou Parisiennes de la bonne société29.

Comme je l'ai montré ailleurs plus en détail, l'orée du $\mathrm{Xx}^{\mathrm{e}}$ siècle voit bien renaître des utopies comme en avaient connu les premières décennies du $\mathrm{XIX}^{\mathrm{e}}$ siècle, mais il s'agit plutôt de «dystopies». L'optimisme rémanent du culte du progrès censé accompagner l'orée d'un nouveau siècle y est largement chargé de menaces et de mauvaises surprises. Toutes s'inspirent en fait d'une vision de l'histoire empreinte des connotations négatives des décennies précédentes ${ }^{30}$.

C'est la Première Guerre mondiale, catastrophe qui dépasse en horreur tous ces pronostics anxiogènes, et la mise sur orbite progressive d'un chrononyme nouveau et concurrent, "Belle époque», qui contribuent le plus à compromettre la carrière et le souvenir de la «fin de siècle». Proust qui a vécu les deux périodes et cherche à rendre la couleur spécifique des «temps perdus» sait indiquer aux lecteurs nostalgiques la spécificité de l'avant 1900 par un réemploi sélectif de cette notion désormais passée de mode. Ainsi dans le passage suivant de Du côté de chez Swann où Mme Cottard, incarnation du goût moyen en peinture, dit à Swann à propos d'un portrait un peu trop avant-gardiste à son avis :

«Mais je dois vous l'avouer franchement, vous ne me trouverez pas très fin de siècle, mais je le dis comme je le pense, je ne comprends pas. Mon Dieu, je reconnais les qualités qu'il y a dans le portrait de mon mari, c'est moins étrange que ce qu'il fait d'habitude, mais il a fallu qu'il lui fasse des moustaches bleues (...) je trouve que la première qualité d'un portrait, surtout quand il coûte 10000 francs, est d'être ressemblant et d'une ressemblance agréable ${ }^{31}$.»

l'histoire à la fiction. Les écrivains français et l'affaire Dreyfus, thèse de littérature, université de Paris 3 Sorbonne nouvelle, 2010, <https://tel.archives-ouvertes.fr/tel-00860862>

29. La genèse du roman s'étale d'ailleurs sur la décennie 1890 : une première version brève en feuilleton paraît en 1891-1892, une seconde version en feuilleton, enrichie de nouveaux épisodes, sort dans la Revue blanche du 15 janvier au $1^{\text {er }}$ juin 1900. Enfin le volume est publié le $1^{\text {er }}$ juillet 1900 chez Fasquelle. Boudé par la critique, le roman connaît le succès parce qu'il a touché là où cela fait mal (cf. Pierre Michel et Jean-François Nivet, Octave Mirbeau l'imprécateur au cour fidèle, biographie, Paris, Séguier, 1990).

30. Christophe Charle, Discordance des temps, op. cit., p. 327-337. Un phénomène analogue se produit en Angleterre : cf. John Stokes, Fin de siècle, Fin du globe. Fears and Fantasies of the Late Nineteenth Century, Londres, Mac Millan, 1992.

31. Marcel Proust, Du côté de chez Swann (1913), in À la recherche du temps perdu, éd. Pierre Clarac et André Ferré, volume I, Paris, Gallimard, Bibliothèque de la Pléiade, 1984, p. 375, cité aussi par Luzius Keller, «Selbstdarstellung, Porträt und Karikatur im Fin de siècle : die Gräfin Castiglione, 
Le lecteur âgé de 1913 a dû sourire en lisant ce passage, les audaces impressionnistes de la fin du XIX ${ }^{\mathrm{e}}$ siècle qui choquaient la femme du docteur Cottard ("moustaches bleues») paraissant bien timides désormais après les rugissements des «Fauves» ou les déformations cubistes des figures humaines proposées au Salon d'automne.

Malgré son côté désuet et affadi une ou deux décennies après 1900, dont témoigne ce réemploi ironique et moqueur par Proust pour faire couleur temporelle, "fin de siècle" va connaître une renaissance fulgurante à un siècle de distance de son émergence. Une interrogation de l'outil de visualisation Ngram viewer proposé par Google books met en évidence le renouveau de la présence de l'expression dans une importante production imprimée à partir des années 1980. La courbe est en parfaite symétrie avec ce qui s'était produit cent ans plus tôt. Il s'agit moins d'ailleurs de désigner les décennies terminales du $\mathrm{Xx}^{e}$ siècle que de revisiter la "vraie» fin de siècle précédente, contribuant à légitimer sa valeur heuristique en privilégiant en réalité dans la plupart des travaux qui s'en occupent une très mince fraction de la société et de la culture qui l'avait vu naître et mise en circulation. Ceci concerne d'ailleurs autant la production en français qu'en anglais ou en allemand où le chrononyme «fin de siècle» français est repris tel quel, non traduit, dans les titres des ouvrages historiques ou littéraires, et se charge de connotations (sophistication, esthétisme, avant-gardisme, modernité exacerbée) puisées dans les thématiques de la fin du XIX ${ }^{e}$ siècle. Mais cette fois elles sont prises de manière positive alors qu'elles étaient le plus souvent dénoncées par les commentateurs et les critiques du siècle précédent. Il n'est pas possible de faire l'exégèse en détail de l'ensemble de cette production proportionnellement plus abondante que celle née dans les dernières décennies du siècle précédent, mais il est incontestable que le réemploi courant de l'expression tient à son parfum d'époque et trouve quelque écho au moment de sa reviviscence, parce qu'elle entre en résonance avec les thématiques post-modernes en plein essor au même moment, sous la plume des spécialistes qui s'en emparent.

Si l'on se limite aux ouvrages où l'expression est nommément citée dans le titre depuis la Deuxième Guerre mondiale conservés à la Library of Congress $^{32}$, la chronologie suggérée par Ngram viewer peut être affinée mais aussi confirmée. On ne dénombre que 3 titres seulement entre 1946 et 1959, 5 entre 1960 et 1969; ensuite la présence de l'expression dans le titre suit une progression géométrique : 12 entre 1970 et 1979, 33 entre 1980 et 1989, 108 dans la décennie 1990. Le mouvement se poursuit depuis puisque le catalogue enregistre encore 96 ouvrages invoquant la «fin de siècle» publiés entre 2008 à 2015.

Robert de Montesquiou, Marcel Proust», in Rainer WARning et Winfried Wehle (dir.), Fin de Siècle, Munich, Wilhelm Fink, 2002, Romanistisches Kolloquium, X, p. 125.

32. J'ai préféré ce catalogue à celui de la BNF parce qu'il couvre mieux la production anglophone et germanophone et possède surtout de meilleures fonctionnalités pour accéder aux contenus des livres et éviter les «faux amis». 
Les deux décennies décisives pour l'expansion du chrononyme peuvent être symbolisées par deux exemples célèbres et les plus précoces contributions savantes sur la "fin de siècle»: Fin de siècle Vienna (1979) de Carl E. Schorske et France fin de siècle d'Eugen Weber (1986) ${ }^{33}$. Pourtant l'ouvrage de Schorske dont la fortune et l'influence ultérieures ne sont plus à démontrer dans de nombreux pays ${ }^{34}$ fait un usage plutôt lâche du chrononyme qu'il utilise pour son titre. L'expression sert surtout à relier 4 articles assez divers parus bien avant dans des revues sur une vingtaine d'années : seul le premier d'entre eux «Politics and the Psyche in Fin-de-siècle Vienna : Schnitzler and Hofmannsthal» mentionne d'ailleurs la catégorie fin de siècle ${ }^{35}$. L'empan chronologique de chacun des essais est très variable, certains vont des années 1860 aux années 1910. Notamment le chapitre II traite en détail la construction de la Ringstrasse et son orientation historiciste puis sa critique par les architectes anti-historicistes, Camillo Sitte et Otto Wagner. Il en va de même du chapitre vi ( «la transformation dans le jardin») qui va des lendemains de la révolution 1848 à la fin du XIX $x^{e}$ siècle. D'autres concernent surtout l'avant 1914 (chapitre VII «Explosion dans le jardin : Kokoschka et Schoenberg»). Deux sont fixés sur le tournant 1900 (le chapitre v sur Gustav Klimt et la Sécession viennoise, le chapitre iv sur l'interprétation des rêves de Freud). L'expression «fin de siècle» ne figure même pas à l'index final et désigne finalement moins une époque fermée sur elle-même que la thèse centrale du livre : la crise du libéralisme fondé sur la croyance au progrès et à la raison a poussé les créateurs ou intellectuels viennois au repli ou à l'élitisme face à un monde de plus en plus hostile où nationalisme, populisme et antisémitisme marginalisent la grande bourgeoisie et les artistes ou écrivains qui lui sont liés. En dépit des liens entre avant-gardes européennes, la "fin de siècle" viennoise, origine de ce que les historiens de la culture anglophone appellent modernism est en réalité bien différente de la parisienne qui a servi à la baptiser, comme l'indique ce passage comparatif :

«In brief, the Austrian aesthetes were neither as alienated from their society as their French soul-mates nor as engaged in it as their English ones. They lacked the bitter anti bourgeois spirit of the first and the warm melioristic thrust of the second. Neither dégagé nor engagé, the Austrian aesthetes were alienated not from their class, but with it from a society that defeated its expectations and rejected its values ${ }^{36} . "$

33. Respectivement Londres, Weidenfeld et Nicholson et Cambridge (Mass) Harvard University Press 1979; traductions françaises respectivement : Paris, Seuil, 1983 et Paris, Fayard, 1986.

34. Lauréat du prix Pulitzer, le livre est traduit en espagnol en 1981 (Barcelone), en allemand en 1982, en français en 1983, en portugais (Brésil) en 1988, en italien en 1991. Plusieurs ouvrages se relient aux problématiques de Schorske en particulier celui de Deborah L. Silverman, Art Nouveau in Fin-de-Siècle France: Politics, Psychology, and Style, Berkeley, University of California Press, 1989 (traduction française Paris, Flammarion, 1994).

35. Première publication American Historical Review, vol. 66, juillet 1961, p. 930-946.

36. Carl Schorske, op. cit., p. 304. 
Ce n'est pas le lieu ici, malgré toute l'admiration qu'on peut avoir pour ce livre pionnier, de critiquer en détail la comparaison assez rhétorique (fondée sur une double négation symétrique un peu trop belle pour rendre compte de champs intellectuels aussi différents et compliqués) entre avant-gardes ou intellectuels des trois pays cités. La symétrie est trop balancée pour emporter l'adhésion et j'ai essayé de montrer ailleurs en détail que l'aliénation invoquée ici de l'avant-garde parisienne n'empêchait nullement son engagement hors des sentiers classiques de la politique lors de l'affaire Dreyfus, tandis que le libéralisme supposé "naturel» des intellectuels anglais pouvait conduire certains à l'art pour l'art, d'autres à la critique du libéralisme pour embrasser le socialisme (les intellectuels fabiens), d'autres au chauvinisme impérial et raciste (Kipling et beaucoup d'autres) ${ }^{37}$. Ce passage illustre surtout que l'expression française transposée à Vienne désigne un tout autre moment social et intellectuel ou artistique que la «fin de siècle» originelle française décrite précédemment.

Aura-t-on plus de succès, comme on pourrait le penser avec l'ouvrage de Weber, Fin de siècle France? Rien n'est moins sûr. Sans doute, les deux premiers chapitres «Décadence» et "Transgressions" sont-ils bien en phase avec l'air du temps «fin-de-siècle» des stéréotypes des deux dernières décennies du $\mathrm{XIX}^{\mathrm{e}}$ siècle, mais presque tous les autres, sauf le chapitre politique («la crise permanente») tentent de relativiser ce qui ne concernait en réalité qu'une très mince fraction des Français. Les chapitres vi à XI du livre et la conclusion "un monde (un peu) meilleur?» regardent plutôt du côté du tableau d'une «belle époque» et d'une avancée du progrès - ainsi le chapitre sur la bicyclette et l'automobile (x) ou celui sur les sports et les activités physiques (xI) - que d'une France du déclin et de la dépression. Même dans les chapitres les plus centrés sur la fin de siècle et sa coloration négative spécifique, Eugen Weber ne cesse d'utiliser des exemples situés hors période (avant ou après) et de relativiser la validité ou la pertinence démonstrative des citations de contemporains, de médecins, d'écrivains ou de faits divers pour nuancer le pessimisme de l'époque :

«Ce qui me frappe c'est ce décalage entre le progrès matériel et la déréliction spirituelle qui évoque tellement notre propre époque. Tant de choses allaient bien, même en France, à la fin du XIX', tandis que l'on disait tant de choses laissant entendre que tout allait $\mathrm{mal}^{38}$.»

37. Cf. Christophe Charle, Naissance des "intellectuels» 1880-1900, Paris, Minuit, 1990; Les Intellectuels en Europe au XIX' siècle, essai d'histoire comparée, Paris, Seuil, 1996; Christophe Charle, Julien Vincent, Jay Winter (eds), Anglo-French Attitudes. Comparisons and Transfers between French and English Intellectuals $18^{\text {th }}-20^{\text {th }}$ Centuries, Manchester, Manchester University Press, 2007; Stefan Collini, Absent Minds. Intellectuals in Britain, Oxford, Oxford University Press, 2005.

38. Eugen Weber, Fin de siècle, la France à la fin du $\mathrm{xIX}^{\mathrm{e}}$ siècle, Paris, Fayard, 1986, p. 9. 
L'allusion à «notre époque» est sans doute la plus importante et explicative de la fortune posthume du terme à cent ans de distance. Au moment où se dissipe l'euphorie relative des années 1960-1970 fondée sur la prospérité générale et l'avènement de la société de consommation dans les pays occidentaux, les multiples transformations des décennies terminales $\mathrm{du} \mathrm{xx}^{\mathrm{e}}$ siècle entrent en résonance avec celles du XIX ${ }^{\mathrm{e}}$ siècle ${ }^{39}$. Spécialistes des avant-gardes, littéraires et comparatistes, historiens de la culture et de l'art, essayistes sur l'air du temps, amateurs de commémoration trouvent ample matière à leurs analyses rétrospectives pour réhabiliter les années 1880-1890. La multiplication des Expositions (notamment Vienne naissance d'un siècle, L'Apocalypse joyeuse au Centre Pompidou en 1986), de nouveaux musées (le musée d'Orsay ouvre ses portes la même année), les rééditions d'ouvrages d'époque dans la collection «fin de siècle» chez 10/18 dirigée par Hubert Juin à partir de 1975, la multiplication des thèses sur les auteurs les plus marginaux (Félix Fénéon ${ }^{40}$, Alfred Jarry ${ }^{41}$, Rachilde ${ }^{42}$ ) élargissent au public cultivé cette nostalgie sélective qu'on transpose même sur des pays où la notion n'a jamais eu cours $^{43}$.

Un chrononyme savant nouveau est né qui renie en grande partie ses origines, mais pour combien de temps?

\section{Christophe Charle est professeur d'histoire contemporaine à l'Université Paris 1 Panthéon-Sorbonne (IHMC) et membre honoraire de l'Institut Universitaire de France} 285.

39. J'ai moi-même esquissé la comparaison dans l'épilogue de Paris fin de siècle, op. cit., p. 275-

40. Joan Ungersma Halperin, Félix Fénéon, Aesthete \& Anarchist in Fin-de-Siècle Paris, New Haven, Yale University Press, 1986.

41. Henri Bordillon [dir.], Alfred Jarry, Centre culturel international de Cerisy-la-Salle (27 août-6 septembre 1981), Paris, P. Belfond, 1985.

42. Michael R. Finn, Hysteria, Hypnotism, the Spirits, and Pornography: Fin-de-Siecle Cultural Discourses in the Decadent Rachilde, Newark, University of Delaware Press, 2009.

43. Voir en dernier lieu la tentative pour réconcilier "histoire globale» et "fin de siècle» dans un ouvrage collectif : Michael Salter (ed.), Fin-de-siècle World, Londres, Routledge, 2015. 